\title{
Acute Motor Axonal Neuropathy
}

National Cancer Institute

\section{Source}

National Cancer Institute. Acute Motor Axonal Neuropathy. NCI Thesaurus. Code

C116929.

A subtype of Guillain-Barre syndrome that targets motor axons, and is characterized by symmetric limb weakness, diffuse areflexia, facial and oropharyngeal muscle weakness, and respiratory insufficiency. 